ISI Impact Factor

(2019-20): 1.628

IC Value (2019): 90.81

$\operatorname{SJIF}(2020)=7.893$

(c) (1) (8)

Copyright@IJCRR

\section{$\nabla$ \\ IJCRR \\ Section: Healthcare \\ Routein Immunization Coverage and Factors Associated with Non-Compliance of Immunizations by Parents of Children Aged 0-5 Years}

\author{
Manisha Praharaj ${ }^{1}$, Kshirabdhi Tanaya ${ }^{1}$, Saurjya Ranjan Das ${ }^{2}$
}

1,2MSc. Tutor, SUM Nursing College, Siksha 'O' Anusandhan (Deemed to be University), Bhubneswar751003, Odisha, India; ${ }^{2}$ Associate Professor, IMS \& SUM Hospital, Siksha 'O' Anusandhan (Deemed to be University), Bhubneswar 751003, Odisha, India.

\title{
ABSTRACT
}

Introduction: Despite many efforts from the government of India, free of cost immunization and ongoing National immunization program, the result of immunization coverage in our country is not satisfactory. The present study is conducted to find out the factors that cause incomplete immunization.

Aims: To assess the factors causing non-compliance of immunization by the parents of 0-5year children. To find out the association between non-compliance of immunization with their selected demographic variable.

Methodology: The present study was carried out as a non-experimental survey. A total of 169 parents of children aged 0 - 5 years were included in the study. The data was collected regarding demographic information, immunization status, doses of vaccines and factors responsible for incomplete immunization by interviewing parents, by dichotomous (yes/no scale) and by checking vaccine card. Validity was checked by experts and reliability was done by test-retest correlation.

Results: The result shows, number of children with complete and partial immunization was $66.27 \%$ and $33.72 \%$ respectively. $25.44 \%$ of parents believe the vaccine is against religion and culture, for $33.13 \%$ of children the barrier of vaccination is the timing of immunization centre, distance or cost to reach immunization centre, $20.11 \%$ skipped vaccine due to lack of vaccines in Immunization centre, $0.07 \%$ believe the vaccine can hurt baby, $48.52 \%$ did not get proper information regarding immunization from health care providers. There was a significant association between age of parents, source of information, education and occupation of parents and non-compliance of immunization.

Conclusion: This study showed that some children have not completed vaccines according to their age. So, health care providers should be more active to motivate the parents to give all immunization in the proper scheduled time.

Key Words: Immunization, Factors, Noncompliance, Parents, Children, Odisha

\section{INTRODUCTION}

Immunization prevents most childhood diseases and it is the most cost-effective way of health intervention. The Government of India implements the vaccination programme by the guidelines set by EPI (Expanded Programme on Immunization). The immunization schedule includes OPV, IPV, BCG, Pentavalent vaccine (Diphtheria, Pertussis, Tetanus, Hib, Hepatitis - B), Rotavirus vaccine, MMR vaccine (measles, mumps, rubella) and Japanese encephalitis vaccine. According to the NFHS-3 in India, the percentage of immunization increased from $36.0 \%$ in (1992) to $42.0 \%$ in (1998) and further to $44.0 \%$ in (2005) of the children in age one to two years. But it is still, very less than the desired goal of achieving $85.0 \%$ coverage. ${ }^{1,2}$ These all vaccines are provided free of cost to all children. The EPI has conducted immunization day and campaigns, fixed areas for vaccinations, mobile vaccination teams to reach small villages and unvaccinated areas. ${ }^{3}$ As per many surveys still there are a lot of children who are not receiving complete recommended vaccines according to their age.

The knowledge and belief of parents, proper information regarding vaccines are more important factors for giving vaccines. ${ }^{4}$ many factors contribute to non-compliance of immunization including difficulties in reaching immunization service centre, lack of awareness, misinformation about vaccines, adverse effect of vaccines, lack of knowledge regarding vaccine-preventable diseases, lack of communication between parents and source of immunization

\section{Corresponding Author:}

Manisha Praharaj, MSc, Tutor, SUM Nursing College, Siksha 'O' Anusandhan (Deemed to be University), Bhubneswar 751003, Odisha, India. Email: manishapraharaj21486@gmail.com

ISSN: 2231-2196 (Print)ＩSSN: 0975-5241 (Online)

Received: $09.11 .2020 \quad$ Revised: 14.01 .2021

Accepted: 27.02 .2021

Published: 22.06 .2021 
providers, deficiency of knowledge in parents regarding contraindication and adverse effect of vaccines. Many parents believe mild illness is associated with immunization; therefore, mild illness also considered as not giving vaccines to their children etc. ${ }^{5,6}$ It is important to improve parent's knowledge regarding adverse effects and benefits of vaccines, national immunization schedule and other information. Proper practice of immunization by parents will reduce many infectious diseases and practice can be improved by correct information and proper communication regarding the risks and benefits of vaccines. There should be a strong source of information. Therefore, all health care providers, media, social media play an important role in improving parent's perceptions of the risks and benefits of vaccines. ${ }^{7,8}$

The Global vaccine action plan 2011-2020 approved in 2012 by the World Health Assembly to achieve $\geq 90 \%$ national coverage of all the vaccines in their countries according to their national immunization schedule by $2020 .{ }^{9}$ According to a report given by $\mathrm{H}$. Pletcher in 2017, in India the population of $0-14$ years of children are about 27.78 percentage. ${ }^{10}$ A survey by National Family Health in 2016 in India shows the percentage of vaccination coverage of children between 12-23 months of age received all recommended vaccines in Odisha $78.6 \%$, Punjab $89.5 \%$, Goa $88.4 \%$, West Bengal $84.4 \%$ and Kerala $82.1 \%$. As per World Health Organization and UNICEF 27 million children and 40 million pregnant women worldwide do not receive routine immunization and around 10 million children under the age of five years die every year and over 27 million infants in the world do not get coverage of full routine immunization. In the developing world, it does not only prevent about 3 million child death per year but also has the potential to avert additional 2 million deaths if immunization programmes are expanded and fully implemented. ${ }^{11}$ According to Global Immunization coverage in 2017 the percentages of received different vaccines by the children are: Hib vaccine is estimated at $72 \%$, Hepatitis B vaccine taken $84 \%$, Measles vaccine taken $85 \%$, Rotavirus taken $28 \%$, Polio vaccine taken $85 \% .^{12}$

\section{MATERIALS \& METHODS}

\section{Research design and setting}

The research design was a non-experimental descriptive survey. The research approach used for the present study was quantitative. The data was collected directly from study subjects by using a dichotomous (yes/no scale).

\section{Sample and Sampling technique}

The samples for the present study were the parents of children between 0 to 5 years and the samples were collected from different community areas of Bhubneswar, Odisha by using the purposive sampling technique. The sample size was calculated by $\left(\mathrm{z}^{2^{*}} \mathrm{p}^{\wedge}\left[1-\mathrm{p}^{\wedge}\right] / \varepsilon 2\right)$ where $\mathrm{z}$ is $(1.96), \varepsilon$ is margin error (7.4) and $\mathrm{p}^{\wedge}$ is population proportion (45.6). thus, the sample size for the present study was 169 .

\section{Inclusion and exclusion criteria}

The parents were chosen who has children less than 5 years, who can read and understand the local language because the parents are responsible to vaccinate their child and the study focused on the factor parents could not vaccinate their child. The parents were excluded from the study who did not meet the criteria.

\section{Methods of data collection}

In this cross-sectional study, a self-administered questionnaire was designed to collect data from participants, to know the factors causing non-compliance of immunization, to measure the variables and to test the hypothesis quantitatively. The self-structured questionnaire has two parts. Part A: contains socio-demographic characteristics of parents aged $0-5$ years of children. Part B: consists of 26 questionnaires about perceived barriers for non - compliance of immunization by parents of $0-5$ years of children. Each item has yes/no options. Data collection was done during June July 2019 after formal written permission from Anganwadi worker of Bharatpur AWC (Anganwadi centre), Anganwadi worker of Adibasi gaon-2 AWC, Medical officer of Urban PHC (Public Health Centre) IRC village. The self-structured questionnaire was given to all the participants followed by an interview and checking the immunization card.

Data were analysed by using SPSS (Statistical Package for the social sciences, 20.0) licensed to the institute. Variables are expressed by frequency, percentage, mean and SD (Standard Deviation). Chi-square test was used to find out the association. $\mathrm{P}<0.05$ was considered as statically significant.

\section{Ethical consideration}

Participation in the present study was voluntary and written informed consent was taken from all the participants after informing the purpose of the study and privacy of participants was ensured. The institution ethical committee approved the study.

\section{RESULTS}

A total of 169 parents participated in the present study. All were present during data collection, so the response rate was $100 \%$. Out of 169 participants, maximum parents $(44.12 \%)$ were between the age of $30-40$ years, $(56.2 \%)$ of participants were female(mother). Only $(20.71 \%)$ of participants completed graduation while $(39.6 \%)$ of family income was 
less than 10,000 . Less than half $(42.01 \%)$ of participants had two children while $(66.7 \%)$ of children were age between 1 -3 years. About half $(50.71 \%)$ of the children were female. Furthermore, it was observed that $(48.67 \%)$ of the participants got information about vaccination from health workers.

As mentioned in table 1, $(33.72 \%)$ of children have not given all vaccines according to their age, a few numbers $(27.21 \%)$ of children skipped vaccines from the given schedule, most (78.69\%) of children started vaccines soon after birth, about a third $(39.64 \%)$ of parents hesitates to give the vaccine to their child.

\section{Table 1: Frequency and percentage of immunization coverage $(\mathrm{N}=169)$}

\begin{tabular}{|c|c|c|c|}
\hline $\begin{array}{l}\text { Immunization cov- } \\
\text { erage }\end{array}$ & Yes/no & Frequency & Percentage \\
\hline \multirow{2}{*}{$\begin{array}{l}\text { Have you given all the } \\
\text { vaccines according to } \\
\text { the age of your child? }\end{array}$} & yes & 112 & 66.27 \\
\hline & No & 57 & 33.72 \\
\hline \multirow{2}{*}{$\begin{array}{l}\text { Did you skip any vac- } \\
\text { cine from the sched- } \\
\text { ule? }\end{array}$} & yes & 123 & 72.78 \\
\hline & No & 46 & 27.21 \\
\hline \multirow{2}{*}{$\begin{array}{l}\text { Have you started vac- } \\
\text { cination soon after the } \\
\text { child's birth? }\end{array}$} & Yes & 133 & 78.69 \\
\hline & No & 36 & 21.3 \\
\hline \multirow{2}{*}{$\begin{array}{l}\text { Have you ever hesi- } \\
\text { tated to get a vaccina- } \\
\text { tion for your child? }\end{array}$} & Yes & 67 & 39.64 \\
\hline & No & 102 & 60.35 \\
\hline \multirow{2}{*}{$\begin{array}{l}\text { Any missed vaccine } \\
\text { compensated further? }\end{array}$} & Yes & 142 & 84.02 \\
\hline & No & 27 & $15 \cdot 97$ \\
\hline
\end{tabular}

Table 2 shows the most common factors causing non-compliance of immunization were parents believe vaccines are not needed if a child born healthy $(59.76 \%)$, the vaccine should not be given as children are very small $(56.80 \%)$. A very few (18.34\%) of parents skipped vaccines as they believe vaccines are made from animal sources, $(25.44 \%)$ of parents believe vaccines are against their religion and custom, $(0.07 \%)$ of parents skipped vaccine as they heard of adverse effects of vaccines. About a half $(48.52 \%)$ of parents did not get proper information regarding vaccines from health care workers, $(20.11 \%)$ of children skipped vaccines due to lack of vaccine in immunization centres and about a third (33.13\%) of children skipped vaccine due to timing of immunization centre, distance or cost to reach immunization centre. About half (43.19\%) of parents perceive any rude or uncomfortable behaviour from the vaccination centre.

Table 2: Factors causing non-compliance of immunization $(\mathrm{N}=169)$

Factors

Do you believe vaccines are safe \&effective for a child?

Do you believe a vaccine is not needed if your child born healthy?

Do you believe the vaccine should not give to your child as they are very small?

Do you think vaccines are against your religion and culture?

Have you refused any vaccines as you believe it has been prepared from an animal source?

Do you think vaccination prevent your child from infectious disease?

Have you heard of any adverse reaction to a vaccine that made you reconsider not getting the vaccine?

Is your immunization centre located nearby your home?

Have you skipped any vaccine due to the timing of the immunization centre, distance or cost to reach the immunization centre?

Have you skipped any vaccine due to the lack of vaccines in the Immunization centre?

Did you perceive any rude or uncomfortable behaviour from the vaccination centre?

Do the leaders, teachers and health workers in your community support vaccination for children?

Does your leader or any health care providers advice or give proper information regarding vaccination?
Yes/no Frequency Percentage

$\begin{array}{ccc}\text { yes } & 127 & 75.14 \\ \text { No } & 42 & 24.85 \\ \text { yes } & 101 & 59.76 \\ \text { No } & 68 & 40.23 \\ & & \\ \text { Yes } & 96 & 56.80 \\ \text { No } & 73 & 43.19 \\ & & \\ & & \\ \text { Yes } & 43 & 25.44 \\ \text { No } & 126 & 74.55 \\ & & \\ \text { Yes } & 31 & 18.34 \\ \text { No } & 138 & 81.65 \\ & & \\ & & \\ \text { Yes } & 83 & 49.11 \\ \text { No } & 86 & 50.88 \\ & & \\ \text { Yes } & 12 & 0.07 \\ \text { No } & 157 & 92.89\end{array}$

$\begin{array}{lll}\text { Yes } & 98 & 57.98\end{array}$

$\begin{array}{lll}\text { No } & 71 & 42.01\end{array}$

$\begin{array}{lll}\text { Yes } & 56 & 33.13\end{array}$

No $\quad 113 \quad 66.86$

$\begin{array}{lll}\text { Yes } & 34 & \mathbf{2 0 . 1 1}\end{array}$

No $\quad 135 \quad 79.88$

$\begin{array}{lll}\text { Yes } & 73 \quad 43.19\end{array}$

No $\quad 96 \quad 56.80$

$\begin{array}{lll}\text { Yes } & 112 & 66.27\end{array}$

$\begin{array}{lll} & \text { No } & 57\end{array}$

$\begin{array}{lll}\text { Yes } & 87 & 51.47 \\ \text { No } & 82 & 48.52\end{array}$


Table 3 shows the association of non-compliance with immunization with the demographic variables. It reveals that the age of parents, sources of knowledge, education and occupation of parents was significantly associated as the chisquare value is less than the p-value $(0.05)$ level of significance. There was no significant association between child gender and non-compliance of immunization.

Table 3: Association of non-compliance of immunization with their selected demographic variables $(\mathrm{N}=169)$

\begin{tabular}{|c|c|c|c|}
\hline Demographic variables & $\begin{array}{c}\text { Chi-square } \\
\text { value }\end{array}$ & Df & $\begin{array}{l}\text { p-value } \\
(<0.05)\end{array}$ \\
\hline Age of parents & 11.883 & 3 & $.008^{*}$ \\
\hline Education of parents & 9.495 & 3 & $.023^{*}$ \\
\hline Occupation of parents & 14.453 & 3 & $.002^{*}$ \\
\hline Child gender & 0.002 & 1 & .965 \\
\hline $\begin{array}{l}\text { Previous source of knowl- } \\
\text { edge }\end{array}$ & 8.053 & 2 & $.018^{*}$ \\
\hline
\end{tabular}

\section{DISCUSSION}

Immunization is very much essential to prevent childhood preventable illness because by vaccination child gets protection from many infectious diseases. It is a simple, costeffective way to protect a child from serious infection. Immunization not only protects the child but also prevents the spread of infections to others. The low immunization rate in children is the biggest concern for healthcare in India. Due to less immunization, many children are at risk of preventable infection during childhood, which further increases the health worker's burden, increase the health care cost of the family, also increase child mortality and morbidity and decrease the quality of life. The present study aimed to find out the factors causing non-compliance of immunization among parents. By identifying the causes, further, it will be possible to implement the nursing interventions. The present study assessed the factors are responsible for non-compliance of immunization and result shows (75.14\%) of parents believe vaccines are safe and effective for the child (66.27\%) given full vaccinations according to their age (72.78\%) skipped few vaccines from the recommended schedule, (33.13\%) skipped vaccines due to vaccination timing of immunization centre, $(20.11 \%)$ skipped vaccine due to lack of vaccine in immunization centre, $(0.07 \%)$ of children skipped vaccine because parents heard about the adverse effect of the vaccine, $(25.44 \%)$ of parents believe that vaccines are against religion and culture.

This was similar to findings of another survey conducted by Alejandro Gracia et al in 2014 on understanding the main barriers to immunization in Colombia to better tailor communication strategies. They included 4802 parents and guardians of children aged less than five years. All most all parents know vaccines have health benefits and 4738(98.7\%) have got vaccination cards for their children. Some factors were identified as barriers of immunization, these are factor related to caregivers $(24.4 \%)$, Vaccinators $(19.7 \%)$, health centres $(18.0 \%)$, the health system $(13.4 \%)$, concerns about adverse events (13.1\%) and cultural and religious beliefs $(11.4 \%) .{ }^{13}$ Another study conducted by K.J.Awosan et al on 2018 on knowledge, attitude and compliance with full immunization of children against vaccine-preventable diseases among pregnant mothers in Sokoto, Nigeria shows that (49.1\%) had knowledge on vaccine-preventable diseases, $(91.8 \%)$ had good knowledge on the national immunization schedule. They also found out (52.6\%) not given vaccine due to disapproval of husband and $(26.3 \%)$ due to lack of immunization. ${ }^{14}$

There was a significant association with the age of parents, sources of knowledge, education and occupation of parents as the chi-square value is less than the p-value $(0.05)$ level of significance. There was no significant association found with child gender.

It was similar to the findings of another study conducted by Vinod Kumar Mugada et al, 2017 a descriptive observational study on knowledge towards childhood immunization among mothers and reasons for incomplete immunization. The data was collected from 377 mothers bearing children aged 3years. The result shows that $115(30.50 \%)$ children were partially immunized and the main reason for incomplete immunization is the unavailability of the vaccine. There was a significant association between gender and immunization ( $\mathrm{p}=0.027)$, mother's education and knowledge score $(\mathrm{p}=0.005)$ and area of residence and knowledge $(\mathrm{p}=0.001)$. There was no association found between immunization status of children and area of residence, birth order, or mother's education..$^{15}$

A study conducted by Umar Yunusa et al. in 2017 on determinants of parental compliance with routine childhood immunization schedule in Nassarawa state, Nigeria includes 387 parents of children aged 12 to 23 months shows that $60.0 \%$ of the parents have less knowledge on immunization. Only $24.5 \%$ of children completed full immunization according to their age. There was a significant association between level of education of parents, number of children and parents' knowledge of immunization with the completion of immunization. ${ }^{16}$

Esohe Konwea is done a study in 2018 on determinants of compliance with child immunization among mothers of children under five years of age in Ekiti state, Nigeria. The result shows $80 \%$ compliance with childhood immunization. They found out mother's knowledge and education is significant to a mother's compliance. The mothers who have a high level of education have a high level of compliance. ${ }^{17}$ 
Choudhary Bhagraj, Solanki S conducted a study with 210 caretakers of children aged 12-23 months on knowledge and practices of immunization and the result shows that BCG was administered to $106(95.49 \%)$ of males and $85(85.85 \%)$ of females, DPT III to $83(74.77 \%)$ of males and $70(70.70 \%)$ of females and Measles to 149 (70.96\%) of the total subjects. The dropout rates in both the gender were observed maximum in BCG to Measles (23.58 in males and 20.00 in females) followed by DPT I to DPT III (16.16 in males to 9.75 in females). Knowledge about the correct age of vaccination for Measles, BCG, DPT, OPV were $26.67 \%, 21.43 \%$, $18.57 \%$ and $12.85 \%$ of the study subjects respectively. Fever (54.28\%), swelling $(74.76 \%)$, redness $(60.95 \%)$ on the thigh after DPT emerged as the main side effects of vaccination. Measles (86.19\%) and Polio (65.24\%) were the most commonly heard diseases, among the vaccine-preventable diseases. ${ }^{18}$

This study has some limitations also. The sample size is small and it covers only a few community areas. Some so many children don't complete all the vaccine according to their age.

\section{CONCLUSION}

The responsibility of immunization is shared by all like community, health care providers, parents who are active participants. Proper and effective communication at different level is essential to improve immunization uptake. ${ }^{19}$ Though immunization is provided free of cost, there are many factors like education of parents, occupation, monthly income, gender, living condition, awareness, religion etc play an important role in coverage of immunization. The present study reveals there are some factors which influence coverage of immunization, $8.28 \%$ believe the vaccine is against religion and culture, for $21.8 \%$ of children the barrier of vaccination are the timing of immunization centre, distance or cost to reach immunization centre, $7.1 \%$ skipped vaccine due to lack of vaccines in Immunization centre, $13.06 \%$ believe the vaccine can hurt your baby if it's given in early stage of life, $21.3 \%$ did not get proper information regarding immunization from health care providers. The immunization rate may increase by efforts of health care providers, policymakers and electronic media by raising the consciousness about the importance of immunization.

\section{ACKNOWLEDGEMENT}

The authors are grateful to all the Anganwadi workers and the participants for their active participation and contribution in completing the study.

Conflicts of interest: There is no conflict of interest
Financial support: Present study did not receive financial support.

\section{REFERENCES}

1. Ministry of Health and Family Welfare Government of India. Introduction, child health, maternal health in National Family Health Survey (NFHS-III). Volume I. International institute for population Science Available at http://www.measuredhs.com/ pubs/pdf/FRIND3/ 00FrontMatter00.pdf. Accessed on 02 December 2015.

2. Angadi M M, Jose AP, Udgiri R, A study of knowledge, attitude and practices on immunization of children in urban slums of Bijapur city. Karnataka, India. J Clin Diag Res.2013;7(12) :2803-2806.

3. Singh S, Vashi Dhava $1 \mathrm{M}$, Barriers and opportunities for improving childhood immunization coverage in slums: A quantitative study. Preven Medic Rep. 2019;14, june:100858.

4. Ferrer R, Klein W. Risk perceptions and health behaviour. Journal of Current Opinion in Psychology. 2015; (5): 85-89.

5. Mbengue MAS, Sarr M, Faye A, Determinants of complete immunization among Senegalese children aged 12-23 months: evidence from the demographic and health survey. BMC Public Health 2017;(17): 630.

6. Sharma S. Immunization coverage in India. Institute of Economic Growth: University of Delhi Enclave. Working paper series no. E/283/2007.

7. Qutaiba B Al-lela, Mohd. Bahari B, Are parents' knowledge and practice regarding immunization related to paediatrics' immunization compliance? A mixed-method studies. BMC Pediat. 2014; (1); 20

8. Jheeta M, Newell J. Childhood vaccination in Africa and Asia: the effect of parents' knowledge and attitude. Bull World Health Organ 2008; 86:419-420.

9. WHO 8.7.2008: the module for mid-level managers: The EPI coverage survey WHO/IVB/08.07.08.Availablefrom:http:// www.who.int/immunization/documents/mlm/en/indx.html. (Last accessed on 2016 Jan 15).

10. Pletcher H, population growth in India 2017. India: population growth from 2007 to 2017 . .

11. United States Agency for International Development, Immunization Programmes for healthy children. Immunization basics. Available from: http://www.immunizationbasics.jsi.com/Accessed 31/12/2011.

12. World Health Organization. Immunization, vaccines and biological. WHO/IVB/18.12: 2018. Available from: .

13. Gracia L DA, Trumbo SP, understanding the main barriers to immunization in Colombia to better tailor communication strategies. BMC public health. 2014; (14):669.

14. Awosan KJ, M.T.O. Ibrahim, Knowledge, attitude and compliance with full immunization of children against vaccine-preventable diseases among pregnant mothers in Sokoto, /Nigeria. Int J Contemp Med Res. 2018;(5):10-16.

15. Mugada V DivyaSai, knowledge towards childhood immunization among mothers \& reasons for incomplete immunization. J Appl Pharm Sci. 2017: 7(10):157-161.

16. Bello L, Yunusa U, Determinants of parental compliance with routine childhood immunization schedule in Nassarawa State, Nigeria. Int J Nur Care. 2017;(1): 1-7

17. Esohe Konwea Patience, Alice D, Determinants of compliance with child immunization among mothers of children under five years of age in Ekiti State, Nigeria. J Health Res. 2018; 3(32). Pp.229-236. 
18. Choudhary B, Solanki S. Knowledge and Practices of Caretakers About Immunization Among Children Aged 12 - 23 Months of Rural block Gudamalani, District Barmer (Rajasthan). Int J Curr Res Rev. 2017;9(3);28-33.
19. Babirye JN, Berwa R, More support for mothers: a qualitative study on factors affecting immunization behaviour in Kampala, Uganda. BMC Public Health. 2011;(11). P.723. 